\title{
Crystal Structure of a Dimeric Oxidized form of Human Peroxiredoxin 5
}

\author{
Christine Evrard ${ }^{1}$, Arnaud Capron ${ }^{1}$, Cécile Marchand ${ }^{2}$, André Clippe ${ }^{2}$ Ruddy Wattiez ${ }^{3}$, Patrice Soumillion ${ }^{4}$, \\ Bernard Knoops ${ }^{2}$ and Jean-Paul Declercq ${ }^{1}$ \\ ${ }^{1}$ Unit of Structural Chemistry (CSTR), Université catholique de Louvain, 1 place Louis Pasteur, B-1348 Louvain-la-Neuve, Belgium \\ ${ }^{2}$ Laboratory of Cell Biology Institut des Sciences de la Vie Université catholique de Louvain, 5 place Croix du Sud B-1348 Louvain-la-Neuve \\ Belgium \\ ${ }^{3}$ Department of Biological Chemistry, Université de Mons-Hainaut, B-7000 Mons Belgium \\ ${ }^{4}$ Laboratory of Biochemistry Institut des Sciences de la Vie Université catholique de Louvain, 1 place Louis Pasteur B-1348 Louvain-la- \\ Neuve Belgium
}

\begin{abstract}
Peroxiredoxin 5 is the last discovered mammalian member of an ubiquitous family of peroxidases widely distributed among prokaryotes and eukaryotes. Mammalian peroxiredoxin 5 has been recently classified as an atypical 2-Cys peroxiredoxin due to the presence of a conserved peroxidatic N-terminal cysteine (Cys47) and an unconserved resolving C-terminal cysteine residue (Cys151) forming an intramolecular disulfide intermediate in the oxidized enzyme. We have recently reported the crystal structure of human peroxiredoxin 5 in its reduced form. Here, a new crystal form of human peroxiredoxin 5 is described at 2.0 Á resolution. The asymmetric unit contains three polypeptide chains. Surprisingly, beside two reduced chains, the third one is oxidized although the enzyme was crystallized under initial reducing conditions in the presence of $1 \mathrm{mM}$ 1,4-dithio-DL-threitol. The oxidized polypeptide chain forms an homo-dimer with a symmetry-related one through intermolecular disulfide bonds between Cys47 and Cys151. The formation of these disulfide bonds is accompanied by the partial unwinding of the N-terminal parts of the $\alpha 2$ helix, which, in the reduced form, contains the peroxidatic Cys 47 and the $\alpha 6$ helix, which is sequentially close to the resolving residue Cys151. In each monomer of the oxidized chain, the $\mathrm{C}$-terminal part including the $\alpha 6$ helix is completely reorganized and is isolated from the rest of the protein on an extended arm. In the oxidized dimer, the arm belonging to the first monomer now appears at the surface of the second subunit and vice versa.
\end{abstract}

Keywords: antioxidant enzyme; peroxiredoxin; thioredoxin fold; thioredoxin peroxidase; crystal structure

Abbreviations used: PRDX, human peroxiredoxin; Prdx, mouse and rat peroxiredoxin; 1hd2, RCSB Protein Data Bank code of the tetragonal form of human PRDX5; ROS, reactive oxygen species; RNS, reactive nitrogen species; MALDI, matrix-assisted laser desorption/ ionization.

\section{INTRODUCTION}

Peroxiredoxins (PRDXs) constitute a family of ubiquitous peroxidases found in all biological kingdoms. ${ }^{1-5}$ PRDXs reduce hydrogen peroxide, alkyl hydroperoxides and peroxynitrite by the use of reducing equivalents derived from thiol-containing donor molecules such as thioredoxin, glutathione, tryparedoxin, alkyl hydroperoxide reductase flavoprotein oxidoreductase component (AhpF) and cyclophilin $\mathrm{A} .{ }^{6-10}$

Six PRDX isoforms have been identified and characterized in mammals. ${ }^{5}$ Mammalian PRDXs appear to act as regulators of hydrogen peroxide-mediated signal transduction. ${ }^{4,11}$ However, the specific subcellular localization of certain PRDXs in organelles that are sources of reactive oxygen species (ROS) or reactive nitrogen species (RNS), their abundance in many different cell types exposed to high levels of ROS and RNS, and findings that specific inhibition of individual PRDXs renders cells more sensitive to $\mathrm{H}_{2} \mathrm{O}_{2}$ toxicity, lipid peroxidation and apoptosis, argue that PRDXs are also important protective antioxidant enzymes in mammals. ${ }^{12-14}$ Moreover, recent reports on $\operatorname{Prdx} 1, \operatorname{Prdx} 2$ and $\operatorname{Prdx} 6$ inactivations by homologous recombination in mice revealed that these members of mammalian PRDXs are indeed essential enzymes involved, in vivo, in the protection of mammalian cells against deleterious oxidations caused by ROS. ${ }^{15-17}$ 
Figure 1. (a) Topological diagram showing the arrangement of the secondary structural elements in PRDX5. The helices are represented as cylinders and the $\beta$ strands as arrows. The beginning and the end of the secondary structural elements are labeled. The green hatching is only present in the reduced chains (A and B) and the green dashes represent the $\alpha 6$ helix in the oxidized chain $C$. The three helices and the four $\beta$-strands belonging to the thioredoxin fold are colored green and red, respectively while the remaining elements are colored yellow, $(b)$ Alignment of the $C^{\alpha}$ atoms of the three independent polypeptide chains with those of lhd2. The graph shows the distance of each $C^{\alpha}$ atom from the corresponding $C$ atom in lhd2. Chain A is black, chain B is red and chain $C$ is green.

(a)
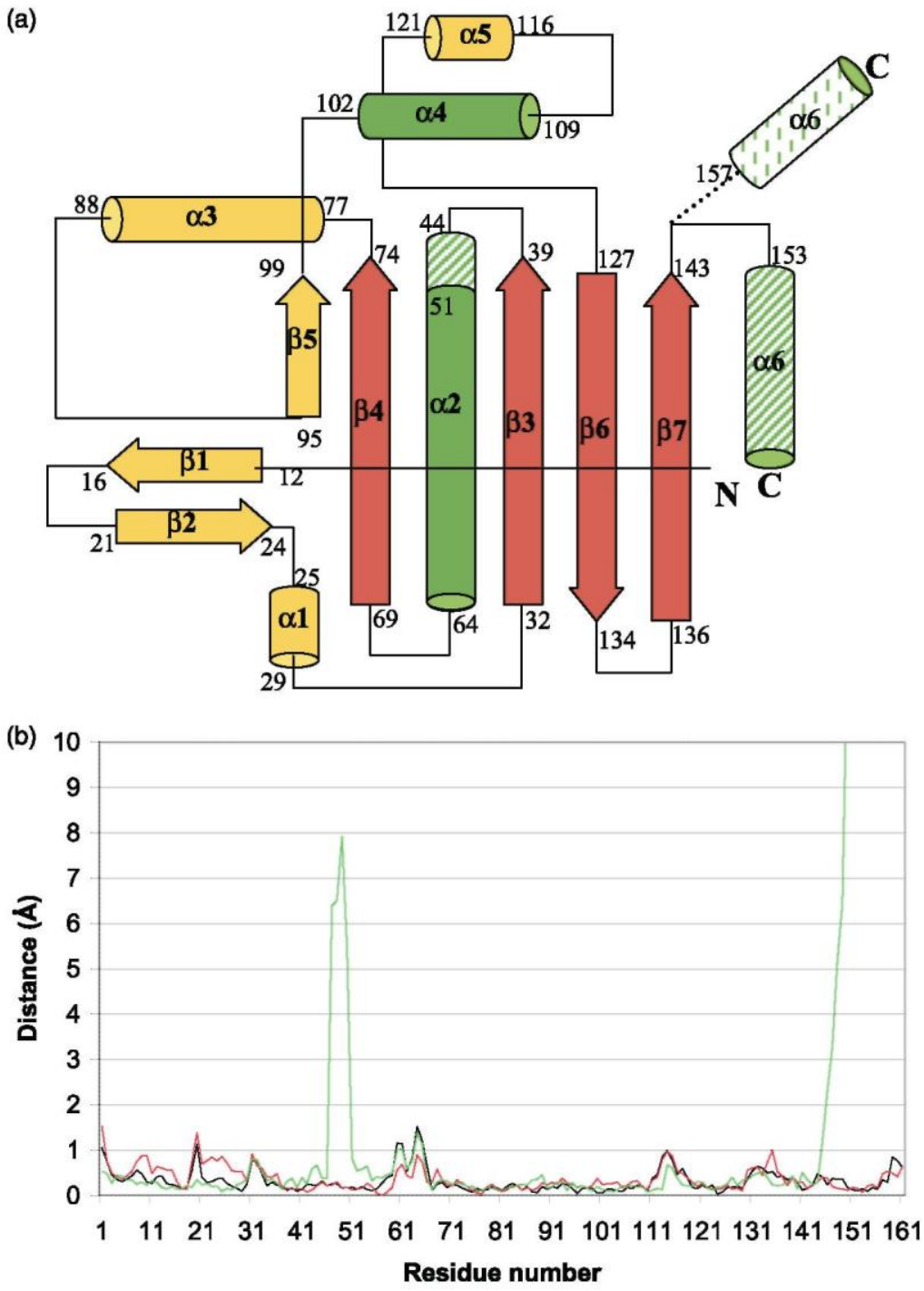

All PRDXs exhibit a conserved peroxidatic cysteine residue in their $\mathrm{N}$-terminal region that attacks the peroxide and is consequently oxidized to cysteine sulfenic acid (Cys-SOH). ${ }^{12}$ Originally, PRDXs were divided into two subgroups, the 1-Cys and the 2-Cys PRDXs, depending on the number of cysteine residues directly involved in catalysis. Mammalian PRDXs are now divided into three subgroups referred to as typical 2-Cys (PRDX1-4), atypical 2-Cys (PRDX5) and 1-Cys (PRDX6) PRDXs. ${ }^{5}$ In the typical 2-Cys subgroup, the second redox-active cysteine, the resolving cysteine, is localized to the $\mathrm{C}$-terminal region of the enzyme in a conserved domain. During peroxidase reaction, the cysteine sulfenic acid from one subunit is attacked by the resolving cysteine of another subunit resulting in the formation of a stable intersubunit disulfide bond which can then be reduced by thioredoxin or cyclophilin to regenerate the enzyme. ${ }^{12,10}$ In atypical 2-Cys PRDXs, the C-terminal resolving cysteine is contained within the same polypeptide chain and the reaction with the peroxidatic cysteine results in the formation of an intramolecular disulfide bond. PRDX5, the only mammalian atypical 2-Cys PRDX identified so far, uses thioredoxin to reduce the disulfide. ${ }^{12}$ Finally, in 1-Cys PRDXs only the N-terminal peroxidatic cysteine is conserved and the resolving cysteine residue is missing. The peroxidatic cysteine sulfenic acid formed upon reaction with peroxides has been reported to be reduced by a thiol-containing reductant such as glutathione or cyclophilin. ${ }^{7,10}$ 
PRDX5, previously named PrxV, AOEB166, PMP20 or ACR1, is the last member of mammalian PRDXs that has been cloned and characterized. ${ }^{12,18-22}$ Human PRDX5 can be addressed to several subcellular compartments such as mitochondria, peroxisomes, the nucleus and the cytosol. ${ }^{12,18-21}$ In a previous report, we presented a crystal structure of human PRDX5 in its reduced form. ${ }^{22}$ The structure revealed that peroxidatic Cys47 and resolving Cys151 were too distant to form an intramolecular disulfide bond upon oxidation without important conformational changes. Also, the enzyme did not form a dimer like other PRDXs (typical 2-Cys and 1-Cys PRDXs) for which dimers are observed in solution and in the crystal, formed by antiparallel association of two $\beta$-strands independent of oxidizing or reducing conditions. ${ }^{5,22}$

Here, we report the crystal structure of a covalent dimeric form of oxidized human PRDX5 at 2.0 Á. Intermolecular disulfide bonds are formed between peroxidatic Cys47 and resolving Cys151 of two different polypeptide chains. However, SDS-PAGE and mass spectrometry analyses show that, upon oxidation, the major form is still the PRDX5 with intramolecular disulfide bond between Cys47 and Cys151. Moreover, we report here that reduced as well as oxidized PRDX5 form contacts which are different from antiparallel association of $\beta$-strands observed in typical 2-Cys and 1-Cys PRDXs. PRDX5 contacts are compatible with the formation of non-covalent dimers in solution.

\section{RESULTS}

\section{Quality of the structure and overall description}

The structure refined at 2.0 Á resolution contains three polypeptide chains labeled A-C in the asymmetric unit. Chains $\mathrm{A}$ and $\mathrm{B}$ are in the reduced form while chain $\mathrm{C}$ is oxidized and forms a dimer by means of disulfide bonds with a symmetry-related chain $\mathrm{C}$. In the three polypeptides, the electron density is well defined along the main chains and for most of the side-chains. It is somewhat more ambiguous at the level of the side-chains of some residues surrounding the disulfide bond in chain $\mathrm{C}$. These regions are also characterized by large thermal motions. The analysis of the Ramachandran plot (not shown) computed with the program PROCHECK ${ }^{23}$ shows that $87.8 \%$ of the non-glycine residues are in the most favored region and there are no residues in disallowed regions. A topological diagram comparing the secondary structures of the three chains is presented in Figure 1(a). In each of them, the overall structure is characterized by the presence of a thioredoxin fold consisting of a four-stranded $\beta$-sheet and three $\alpha$-helices ${ }^{24}$ although in the third chain $(\mathrm{C})$, the last helix $(\alpha 6)$ is no longer flanking the $\beta$-sheet, since it is completely displaced from its usual position in a thioredoxin fold. Each polypeptide chain comprises three additional $\alpha$-helices and three $\beta$-strands, one of which is associated with the thioredoxin $\beta$-sheet to form a fifth strand, while the two remaining ones form an additional two-stranded $\beta$-sheet in the N-terminal part of the chain.

\section{SDS-PAGE analysis}

\section{SDS-PAGE analysis of recombinant PRDX5}

oxidized with increasing concentration of $\mathrm{H}_{2} \mathrm{O}_{2}$ (Figure 2(a)) or reduced by increasing concentration of DTT (Figure 2(b)) showed that the denatured recombinant protein may exist as monomeric (Mox) and dimeric (Dox) oxidized forms as well as monomeric reduced form (Mred). However, the dimeric oxidized form appears always as a minor band compared to monomeric oxidized PRDX5 even after exposure to a high concentration of $\mathrm{H}_{2} \mathrm{O}_{2}$ $(500 \mu \mathrm{M})$. SDS-PAGE analysis established that recombinant PRDX5 is isolated in the oxidized form, since the protein migrates as a minor oxidized dimeric form and a major monomeric oxidized form without prior oxidation by $\mathrm{H}_{2} \mathrm{O}_{2}$ (Figure 2(a) and (b)). As the DTT concentration was increased, the minor dimeric oxidized form decreased from $1 \mathrm{mM}$ DTT to disappear completely at $10 \mathrm{mM}$ and $100 \mathrm{mM}$ DTT. However, as $\mathrm{H}_{2} \mathrm{O}_{2}$ used to oxidize PRDX5 was not removed prior to adding the reductant, part of the DTT is likely oxidized by the excess of $\mathrm{H}_{2} \mathrm{O}_{2}$. Concomitantly, a small upward shift in the migration of the monomeric form is observed upon reduction (Figure 2(b)), consistent with intramolecular disulfide bond reduction as confirmed by mass spectrometry analysis (see below and Figure 2(c)). Also, the use of $N$-ethylmaleimide $(10 \mathrm{mM})$ to block free thiol groups in oxidized PRDX5 indicated that no detectable thiol-disulfide interchange occured during protein denaturation for SDS-PAGE analysis (data not shown).

\section{Gel filtration analysis}

Analysis of recombinant PRDX5 on a gel filtration column with or without $10 \mathrm{mM}$ DTT yielded a major peak with maxima at identical retention times and consistent with a dimeric $38 \mathrm{kDa}$ protein (Figure 2(d)). However, for the oxidized form, an asymmetrical peak with an extended tail on the low molecular mass side is observed. 
This suggests that, under native conditions, the oxidized protein is mainly a dimer but in rapid equilibrium with a minor monomeric form. Gel filtration analyses carried out under reducing or non-reducing conditions using PRDX5 for which free thiol groups were blocked by $N$-ethylmaleimide $(10 \mathrm{mM})$, showed a major peak consistent with a dimeric $38 \mathrm{kDa}$ protein (data not shown).

\section{Mass spectrometry analysis}

Mass spectrometry analysis of the tryptic fragments of monomeric (Mox) and dimeric (Dox) oxidized forms as well as monomeric reduced form (Mred) of recombinant PRDX5, confirmed the existence of a disulfide bond between Cys47 and Cys151 in Mox and Dox forms. Indeed, the matrix-assisted laser desorption/ionization (MALDI)-time-of-flight analysis of the tryptic fragments of Mox and Dox forms showed the presence of two ions $[\mathrm{M}+\mathrm{H}+] \quad 3860 \bullet 0134$ and $3988 \cdot 1089$ corresponding, respectively, to the following peptides linked by a disulfide bridge (Cys47-Cys151): GVLFGVPGAFTPGCys ${ }^{47}$ SK and ALNVEPDGTGL

TTCys ${ }^{151}$ SLAPNIISQL, KGVLFGVPGAFTPGCys ${ }^{47}$ SK and ALN VEPDGTGLTTCys ${ }^{151}$ SLAPNIISQL (Figure 2(c)). Moreover, these ions were not present after reduction and carboxymethylation of the tryptic peptides of the Mox and Dox forms of PRDX5 (data not shown). A minor ion $\left(\left[\mathrm{M}+\mathrm{H}^{+}\right]\right.$4530 2871 ) corresponding to the peptides linked by the disulfide bridge (Cys72-Cys151) was also observed. In contrast, the MALDI-time-offlight analysis of the Mred form showed the presence of ions corresponding to reduced peptides $\left(\left[\mathrm{M}+\mathrm{H}^{+}\right]\right.$ $1664 \cdot 8828,2207 \cdot 0913$ and $2326 \bullet 1958)$. 
Figure 2. SDS-PAGE analysis of PRDX5 under (a) oxidizing or (b) reducing conditions, (c) Analysis by mass spectrometry of tryptic peptides of reduced and oxidized PRDX5 forms examined in (a) and (b). (a) PRDX5 was incubated with various concentrations of $\mathrm{H}_{2} \mathrm{O}_{2}$ and subjected to non-reducing SDS-PAGE. Major monomeric (Mox) and minor dimeric (Dox) oxidized forms of PRDX5 are indicated by arrows. In (b), PRDX5 was first incubated with $500 \mu \mathrm{M} \mathrm{H} \mathrm{H}_{2}$ and then subjected to increasing concentrations of DTT. Monomeric (Mox) and dimeric (Dox) oxidized forms as well as monomeric reduced form (Mred) are indicated by arrows. In (c) mass spectrometric analysis of the tryptic fragments of Mox, Dox and Mred confirmed the existence of a disulfide bond between Cys47 and Cys151 in Mox and Dox. (d) Gel filtration analysis of recombinant PRDX5 under non-reducing and reducing conditions with $10 \mathrm{mM}$ DTT. For both conditions, the protein retention times correspond to a molecular mass of $36 \mathrm{kDa}$. Under non-reducing conditions, an asymmetrical peak is observed, suggesting a rapid equilibrium between a dimeric and a monomeric form.

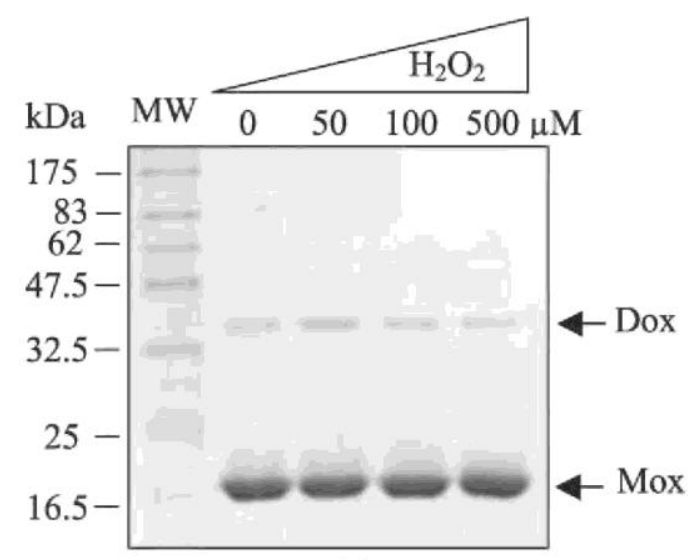

(a)

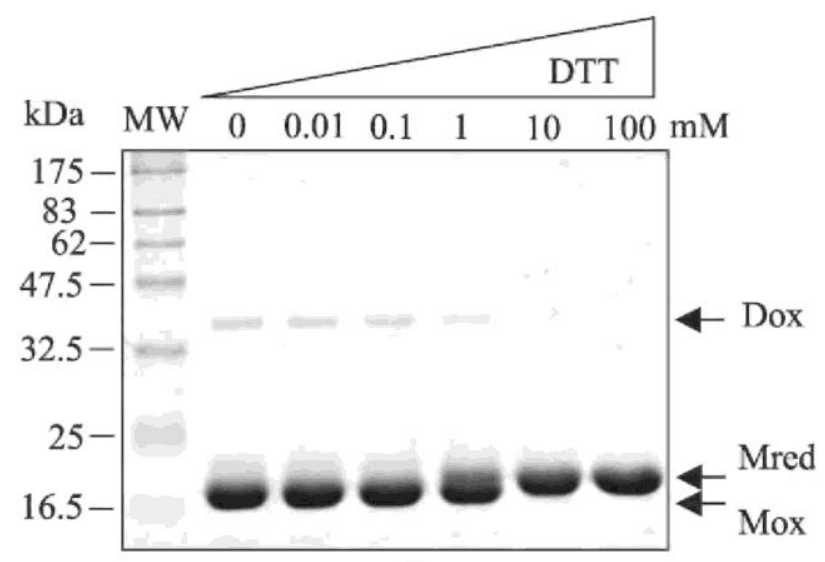

(b)

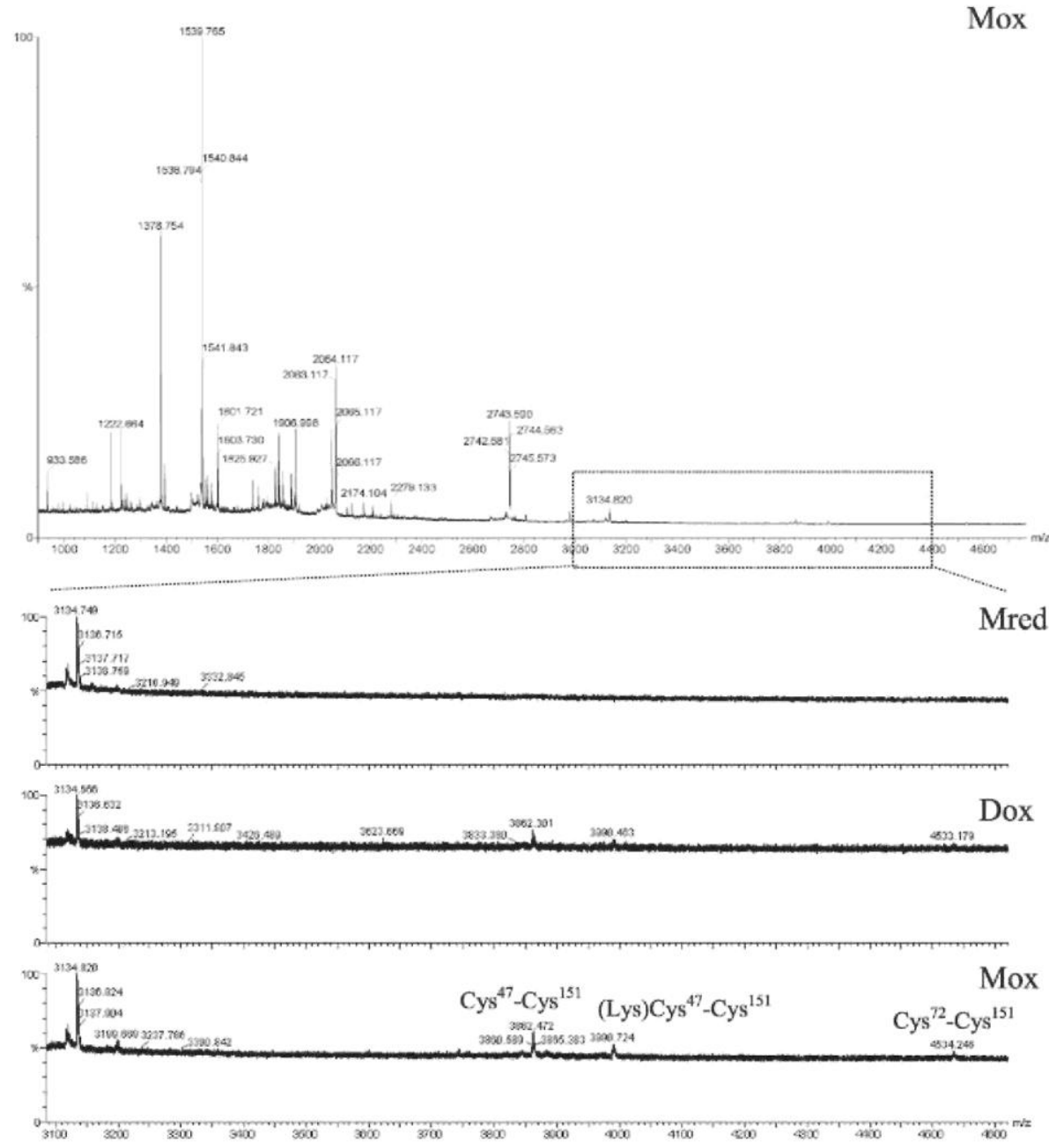

(c) 
Published in: Journal of Molecular Biology (2004), vol. 337, pp. 1079-1090

Status: Postprint (Author's version)

\section{DTT $10 \mathrm{mM}$}

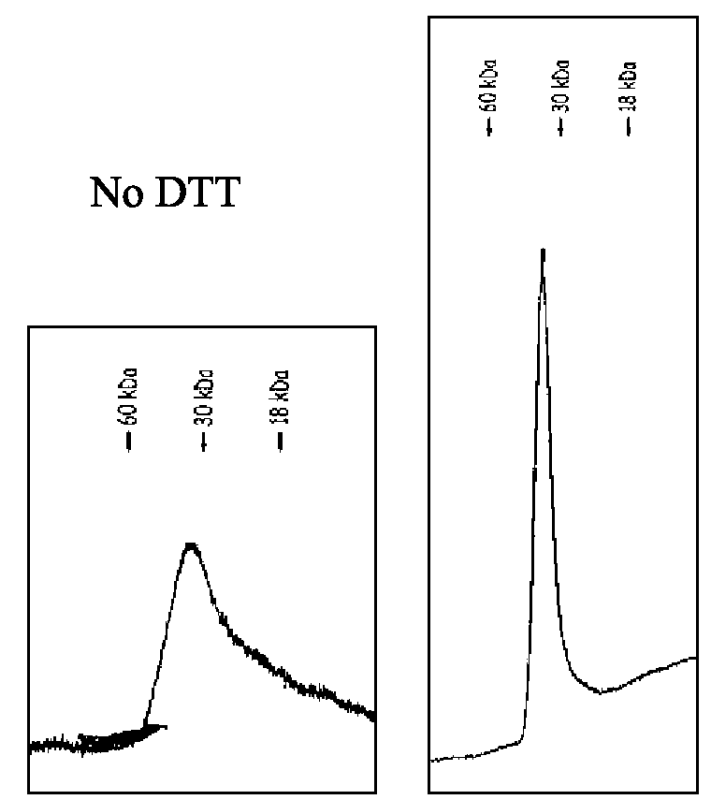

(d) 
Figure 3. (a) and (b) Ribbon diagrams showing the overall organization of the new structure of PRDX5, colored as for Figure 1(a). The reduced chain $A$ is in $(a)$ and the oxidized chain $C$ in $(b) ;$ they are presented in the same orientation. The side-chains of the two Cys residues involved in the catalytic mechanism are represented as balls and sticks. This Figure was prepared using MOLSCRIPT ${ }^{41}$ and Raster $3 D .^{42}$
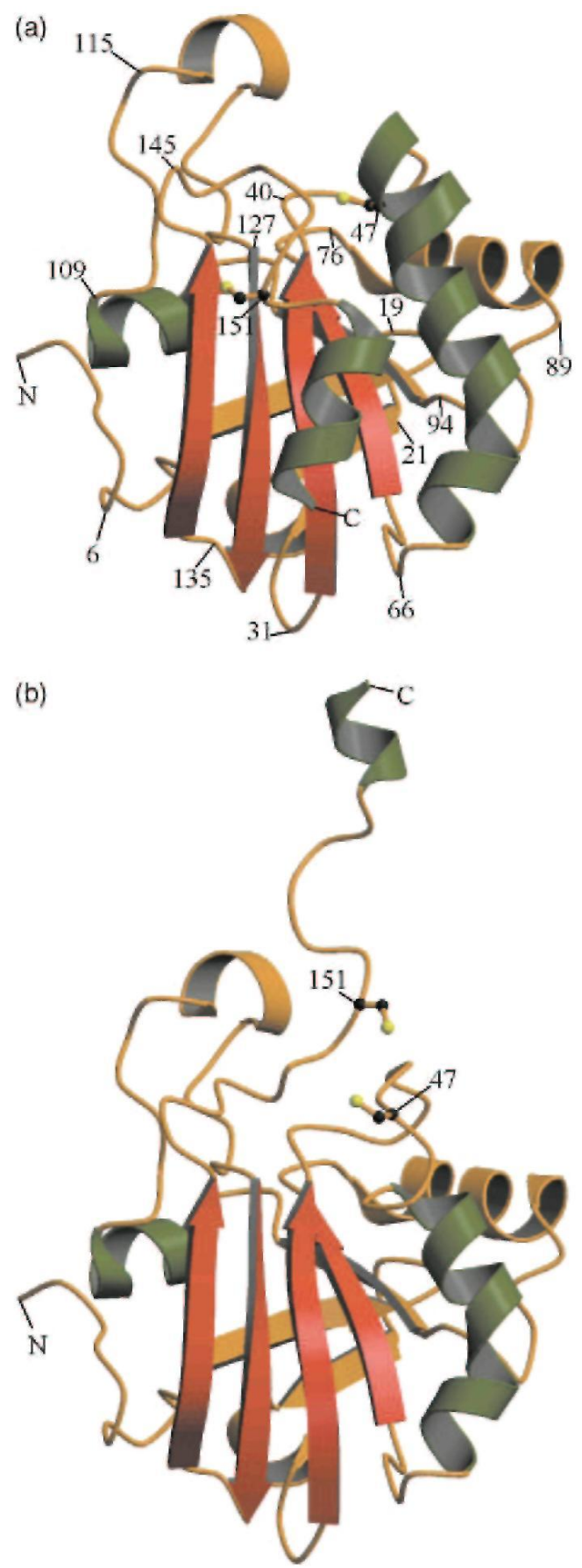

\section{DISCUSSION}

\section{Comparison of reduced and oxidized forms of PRDX5 monomers}

The $161 \mathrm{C}^{\alpha}$ atoms of the three polypeptide chains $\mathrm{A}, \mathrm{B}$ and $\mathrm{C}$ were aligned with those of the previously determined structure $1 \mathrm{hd} 2 .^{22}$ The results are presented in Figure 1(b). The RMS deviation for chains A and B is very small, $0.406 \mathrm{~A}$ and $0.444 \AA$, respectively, while it is as large as $8.565 \AA$ for chain $\mathrm{C}$. For this chain, the largest differences occur in the regions containing the Cys residues (47 and 151) involved in the formation of inter-molecular disulfide bridges. An alignment of chain $\mathrm{C}$ without residues 47-50 and 146-161 reduces the RMS 
deviation to 0.391 Á. Figure 3(a) shows ribbon diagrams comparing chains A and C in the same orientation. Since it appears that the overall folding of the reduced chains A and B is extremely similar to the one described for the tetragonal (pdb:1hd2) crystal form of PRDX5, ${ }^{22}$ it will not be discussed further but will be used for a detailed comparison with chain $\mathrm{C}$.

The first important reorganization observed in the oxidized chain $\mathrm{C}$ concerns the region involving the peroxidatic residue Cys47. In the reduced structure, this residue is part of the kinked $\alpha 2$ helix and it is located in a positively charged pocket exposed to the solvent. Moreover, the sulfur atom of Cys47 is in close interaction with the $\mathrm{N}^{\eta 1}$ atom of Arg127, which is responsible for the positive charge. It is also in contact with the $\mathrm{O}^{\gamma 1}$ atom of Thr44 located at the $\mathrm{N}$-terminal part of the $\alpha 2$ helix and with an oxygen atom of a benzoate ion which restricts the access to the cavity containing Cys $47 .{ }^{22}$ In the oxidized chain $\mathrm{C}$, a large fragment of the N-terminal part of the $\alpha 2$ helix (residues 44-50) is completely unwound. Even if the residues 44-46 still show some helical appearance superimposable on the same residues of the reduced form, this short fragment does no longer belong to the $\alpha 2$ helix in the oxidized chain C. It is worth noting that the kinked part of this helix is located at the C-terminal end and is not concerned with the unwinding observed in the oxidized chain. The peroxidatic residue Cys 47 now appears in a large loop (residues 39-50) and is completely exposed if one considers only one monomer. Also,

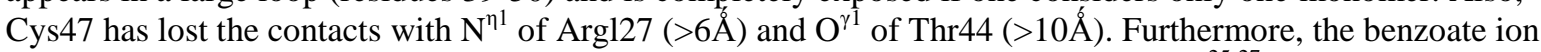
has completely disappeared. The unwinding of the active-site helix has been described ${ }^{25-27}$ in typical 2-Cys PRDX structures, and is thought to play an important role in the disulfide bond formation between the peroxidatic and resolving cysteine residues. In hORF6 ${ }^{28}$ (human PRDX6, member of the 1-Cys PRDX subgroup), the peroxidatic oxidized cysteine Cys47 exists as a reactive cysteine sulfenic acid. It is located at the N-terminal part of the $\alpha 2$ helix and is not involved in a disulfide bond. On the other hand, in HBP23 ${ }^{25}(\operatorname{rat} \operatorname{Prdx} 1$, member of the typical 2-Cys PRDX subgroup) the $\alpha 2$ helix is unwound at its N-terminal end, which contains the corresponding peroxidatic residue Cys52. The latter forms a disulfide bond with the resolving cysteine, Cys173, of another polypeptide chain resulting in the formation of a dimer between these two chains. In the case of TPx$\mathrm{B}^{26}$ (human PRDX2, member of the typical 2-Cys subgroup), the peroxidatic residue is oxidized to sulfinic acid $\left(\mathrm{Cys}-\mathrm{SO}_{2} \mathrm{H}\right)$, an inactive form impeding the formation of a disulfide bond and remains in the wound $\alpha$ helix. The recently solved crystal structure of the Haemophilus influenza hybrid-Prx $5^{27}$ (hyPrx5) also supports the idea that the unwound structure would be important in disulfide bond formation. Indeed, two independent polypeptide chains are present in the asymmetric unit of hyPrx5. In one of these independent chains, the wound conformation of the active-site helix is conserved whilst in the other this helix is unwound at its N-terminal part. This conformational change shortens the distance between the peroxidatic cysteine, Cys49, which becomes a part of a loop, and the resolving cysteine, Cys180, of another sub-unit, bringing the two cysteine residues into the distance needed for disulfide bond formation.

The second region undergoing important rearrangements in the oxidized form of human PRDX5 is in the surroundings of resolving Cys 151. In the reduced form, this residue is located in the loop between the $\beta 7$ strand and the $\alpha 6$ helix and it is well exposed to the exterior. The $\alpha 6$ helix, which immediately follows is folded at the surface of the protein and covers the $\beta 6$ and $\beta 7$ strands in a direction more or less perpendicular to the strands, giving rise to a salt-bridge between $\operatorname{Gln} 160(\alpha 6)$ and Asn141 ( $\beta 7)$, as well as many hydrophobic contacts with the $\alpha 2$ helix and the $\beta 6$ strand. These contacts involve on one side Ilel57, Ile158 and Leu161 in the $\alpha 6$ helix, and on the other side Leu62, Val67 and Val131. In the oxidized form, the situation is completely different and large modifications begin at residue 145 in the loop following the $C$-terminal end of the $\beta 7$ strand. As shown in Figure $3(\mathrm{~b})$, the loop between the $\beta 7$ strand and the $\alpha 6$ helix is completely reversed and brings the remaining part of the protein chain towards the exterior. The $\mathrm{N}$-terminal part of the $\alpha 6$ helix is also unwound for about four residues. This helix appears now at the end of an extended arm and is completely isolated from the rest of the protein. The intramolecular contacts described above for the reduced chain have disappeared and the hydrophobic residues involved in these contacts are exposed. Such a situation would be completely unrealistic if this part of the polypeptide was not incorporated in the formation of a dimer (see below). 
Figure 4. (a) Ribbon diagram of the dimer composed of two oxidized chains $C$ colored green and red. The Cys residues taking part in the two intermolecular disulfide bonds are shown. The C-terminal $\alpha 6$ helix of one subunit comes into close contact with the $\alpha 2$ helix and the $\beta 6$ strand of the other subunit. (b) Molecular surface of the dimer colored according to the local electrostatic potential, ranging from blue (the most positive region) to red (the most negative) and presented in the same orientation as for (a). The positions of the Cys residues are indicated, $(c)$ and $(d)$ Speculation of the possible conformation of an oxidized monomer forming an intramolecular disulfide bond by refolding the extended arm shown in (a) over the same subunit in such a way that it takes exactly the place of the arm of the second subunit. Part (c) is oriented like part (a), and part (d) is oriented like Figure 3(a) and (b). (a), (c) and (d) Prepared using MOLSCRIPT ${ }^{41}$ and Raster3D; ${ }^{42}(b)$ prepared using GRASP ${ }^{43}$

(a)

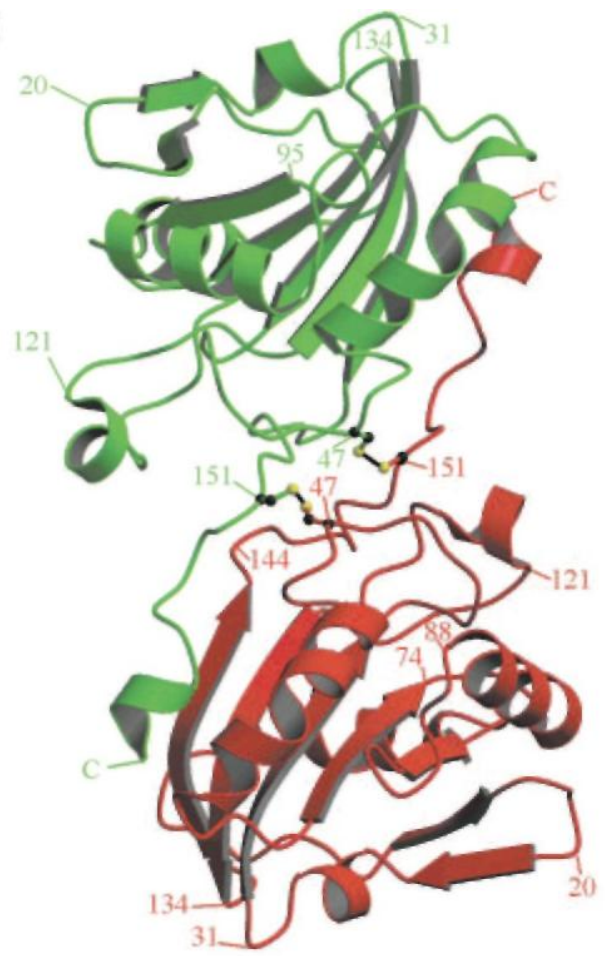

(c)

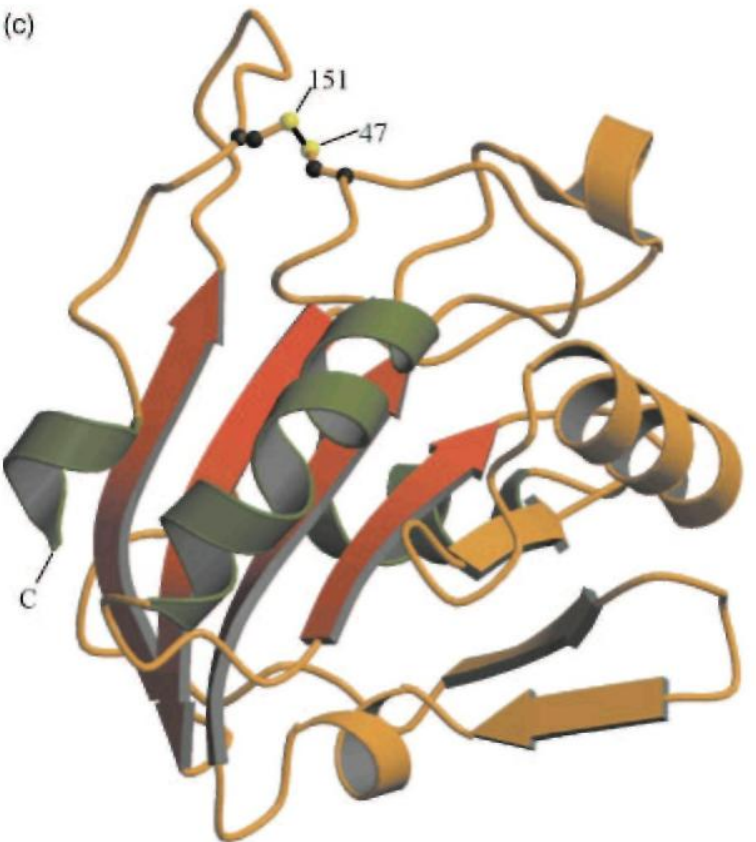

(b)

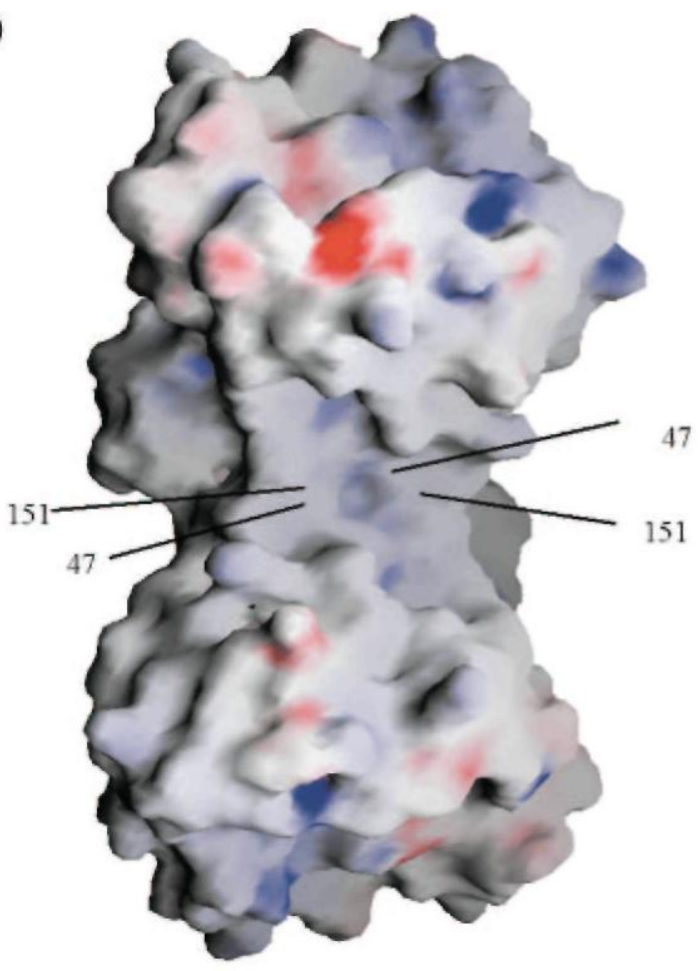

(d)

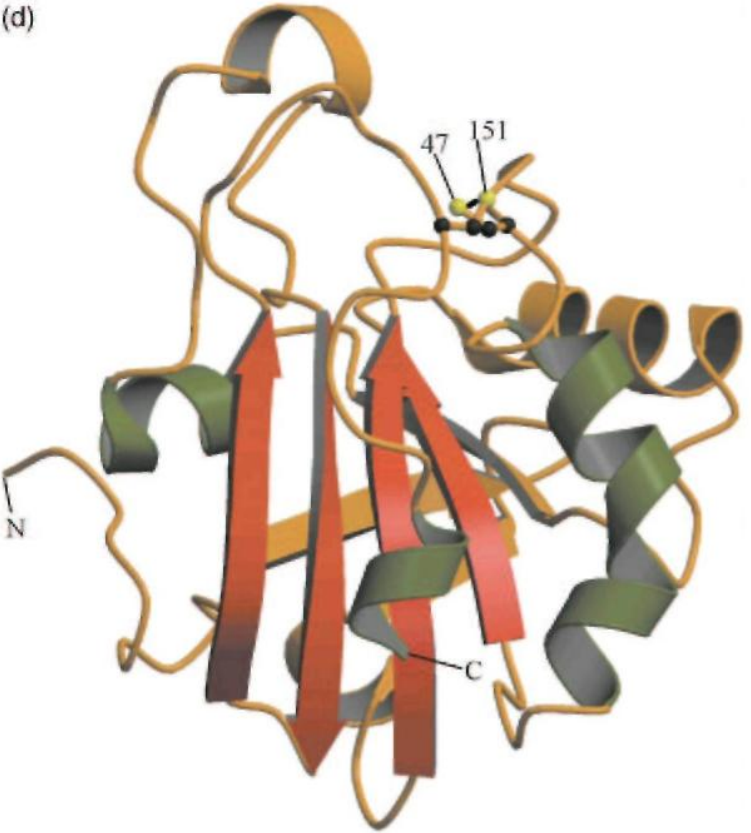




\section{The dimeric oxidized form}

The presence of an oxidized form of PRDX5 was rather unexpected, since the crystallization was performed under reducing conditions in the presence of $1 \mathrm{mM}$ 1,4-dithio-DL-threitol (DTT). Nevertheless, DTT is known to be rapidly oxidized in solution and the redox conditions are thus changing during the time for crystallization.

It was proposed ${ }^{12}$ that, upon oxidation, PRDX5 would form intramolecular disulfide intermediates and is thus monomeric from this point of view. In spite of these biochemical data, the oxidized chain $\mathrm{C}$ forms a covalent dimer with a chain related by a 2-fold crystallographic axis. As shown in Figure 4(a), disulfide bonds are formed between Cys47 of one polypeptide chain and Cys151 of the symmetry related one, and vice versa. In this way, the $\alpha 6$ helix of one chain, at the end of its extended arm, comes in contact with the symmetry-related chain and regenerates in an intermolecular way exactly the same hydrophobic contacts with $\alpha 2$ and $\beta 6$ which were observed in the reduced form in an intramolecular way. The hydrophobic residues are no longer exposed and even the Gln160-Asn141 salt-bridge reappears in the oxidized form. However, this salt-bridge is also intermolecular instead of intramolecular in the reduced chains. The two disulfide bonds of the dimer are very close to each other. They are located at the surface of a kind of widely opened cavity illustrated in Figure 4(b), which appears at the interface between the two monomers.

The picture is becoming even more complicated as the gel filtration analysis indicates a probable rapid equilibrium between a monomeric and a dimeric form under native non-reducing conditions. Although, there is no clue to determine the covalent or non-covalent nature of this dimer, a simplistic one-state model of the oxidized enzyme could be misleading in the way for describing the catalytic mechanism of PRDX5.

\section{Proposed conformation of PRDX5 forming an intramolecular disulfide bond upon oxidation}

The comparison of the monomer shown in Figure 3(b) and the dimer shown in Figure 4(a) as well as SDS-PAGE and mass spectrometry analysis (Figure 2) suggest that the conformation of the oxidized monomeric form, that is based on biochemical data and expected in the catalytic mechanism, ${ }^{12}$ could be created by refolding the extended arm appearing in Figure 3(b) over the very same polypeptide chain in order to regenerate exactly the same contacts observed in the dimer (Figure 4(a)), including the Cys47-Cys151 disulfide bond, but in an intramolecular way. Compared to chain C, this reorganization would only involve two residues (146-147) and the results of these speculations are shown in Figure 4(c) and (d). In this simulation, the peroxidatic Cys47 remains in the unwound part of the $\alpha 2$ helix and this unwinding appears thus to be essential in the disulfide bond formation with the resolving Cys151 either in an intramolecular way or in an intermolecular way. As shown in Figure 4(c) and (d), the intramolecular disulfide bond appearing in this simulation between Cys47 and Cys151 would be accessible for reduction by thioredoxin according to the proposed mechanism. ${ }^{12}$

\section{The packing of the polypeptide chains in comparison with other PRDX structures}

Crystal structures are available for the typical 2-Cys PRDXs, ${ }^{25,26,29,30}$ for one atypical 2-Cys PRDX; ${ }^{.22,31}$ for one hybrid PRDX $5^{27}$ related to the previous category and for one 1-Cys PRDX. ${ }^{28}$ The typical 2-Cys PRDX and the 1-Cys PRDX subgroups are characterized by the formation of a dimer, which is observed independently of the reduced or oxidized state of the protein. This dimer is formed by the antiparallel association of two $\beta$-strands ( $\beta 7$ of each monomer) belonging to the thioredoxin fold, resulting in a ten-stranded (or more) $\beta$-sheet in the dimer. This kind of dimmer is not observed in the known structures of atypical 2-Cys PRDX (PRDX5) and the present study confirms the absence of such dimers in the reduced as well as in the oxidized forms of PRDX5. As shown in Figure 4(a), the dimerization of the oxidized PRDX5 chains by formation of two intermolecular disulfide bonds does not at all associate the $\beta$-sheets of the two monomers. Consequently, if the dimers observed in typical 2-Cys PRDXs do not exist in atypical 2-Cys PRDXs, the decamer formed by association of five dimers ${ }^{26,29,30}$ in typical 2-Cys PRDXs and stabilized by the reduced form of the active-site disulfide ${ }^{5}$ is also non-existent. 
In the crystal structure of hyPrx $5,{ }^{27}$ the existence of PRDX-PRDX dimers was discovered and the authors pointed out that the same contacts exist in the human PRDX5 structure, 1 hd $2 .^{22}$ The contacts involve residues in the $\beta 3-\alpha 2$ loop (residues 43-45), in the a3 helix (residues 79-81), in the $\beta 5$-a4 loop (residues 100-101) and in the $\alpha 5$ helix (residues 117-120) which is characteristic of PRDX5, since it is not observed in other PRDXs structures. We also observe the same non-covalent dimerization in this new structure of PRDX5. The reduced chain A is in contact with a symmetry-related (2-fold axis) chain A (Figure 5(a)), while the reduced chain B is in contact with the oxidized chain C (Figure 5(b)). In each case, the salt-bridge Arg124-Asp77 described in hyPrx $5^{27}$ is also present. The other contacts are mainly hydrophobic and it is worth noting that intermolecular Phe43-Phe43 contacts are present in the interaction between two reduced chains as well as between an oxidized chain and a reduced one, in spite of the localization of this residue in one of the region undergoing important reorganizations in the oxidized form. This non-covalent interaction may thus be present under oxidizing conditions and be responsible of the dimeric form observed by gel filtration (Figure 2(d)). Interestingly, the four contacts described above (the $\beta 3-\alpha 2$ loop, the $\alpha 3$ helix, the $\beta 5-\alpha 4$ loop, the $\alpha 5$ helix) exactly correspond to the four interfacial regions labelled I-IV in the formation of $\left(\alpha_{2}\right)_{5}$ decamers in the subgroup of classical 2-Cys PRDX. ${ }^{5,30}$ Even the $\alpha 5$ helix, which is present only in PRDX5 (atypical 2-Cys PRDX), appears as a kind of inclusion in the region IV of typical 2-Cys PRDXs. It was noticed that the decamer formation was favored in the reduced state and that the oxidized decameric structure of $\mathrm{AhpC}^{30}$ corresponded to a metastable oligomerization intermediate whose presence could be due to the high protein concentration during crystallization. It is very possible that the same kind of metastable association appears when an oxidized chain of PRDX5 is involved in these non-covalent contacts and that the resulting instability could explain the probable rapid equilibrium between a monomeric and a dimeric form in native non-reducing conditions suggested by the gel filtration analysis.

On the other hand, since the oxidized chain $\mathrm{C}$ is also linked to another symmetry-related chain $\mathrm{C}$ by disulfide bonds, tetramers (Figure 5(b)) composed of chains B:C:C:B may appear and this oligomerization, which occurs only in presence of the covalent dimeric oxidized PRDX5, is thus redox-dependent.

Table 1. Data collection and refinement statistics

\begin{tabular}{ll}
\hline Wavelength $(\AA)$ & 0.8441 \\
Resolution range $(\AA)$ & \\
Overall (ov) & $40.0-2.0$ \\
Highest shell (hs) & $2.05-2.0$ \\
Reflections & 164,737 \\
$\quad$ Total & 38,821 \\
$\quad$ Unique & $96.9 / 95.2$ \\
Completeness (\%) (ov/hs) & 0.047 \\
$R_{\text {merge }}$ & \\
Number of non-hydrogen atoms used in refinement & \\
$\quad$ Protein atoms & 3576 \\
$\quad$ Heterogen atoms & 18 \\
Solvent atoms & 62 \\
$R$-factor (ov/hs) & $0.221 / 0.286$ \\
$R_{\text {free }}($ ov/hs) & $0.259 / 0.355$ \\
RMS deviation from ideality & \\
Bonds $(\AA)$ & 0.021 \\
Angles (deg.) & 1.9 \\
Estimated overall coordinate error $(\AA)$ & \\
Based on $R_{\text {free }}$ & 0.168 \\
Based on maximum likelihood & 0.175 \\
\hline
\end{tabular}


Figure 5. (a) Ribbon diagram of the non-covalent dimer between the reduced chain A and a symmetry related chain A. The projection is along the crystallographic 2-fold axis relating the two chains. The models are progressively colored from blue ( $N$ terminus) to red ( $C$ terminus). The regions involved in the contacts are labeled, (b) Ribbon diagram colored as in (a) of the tetramer formed by the association of chains $B($ reduced): $C$ (oxidized):C(oxidized):B(reduced). The B:C and $C: B$ contacts are non-covalent and similar to those observed between the two reduced chains A; the C:C contacts involve two intermolecular disulfide bonds. The side-chains of the peroxidatic and resolving Cys residues are shown. The projection is along the crystallographic 2-fold axis relating the two C chains. This Figure was prepared using MOLSCRIPT ${ }^{41}$ and Raster $3 D .^{42}$
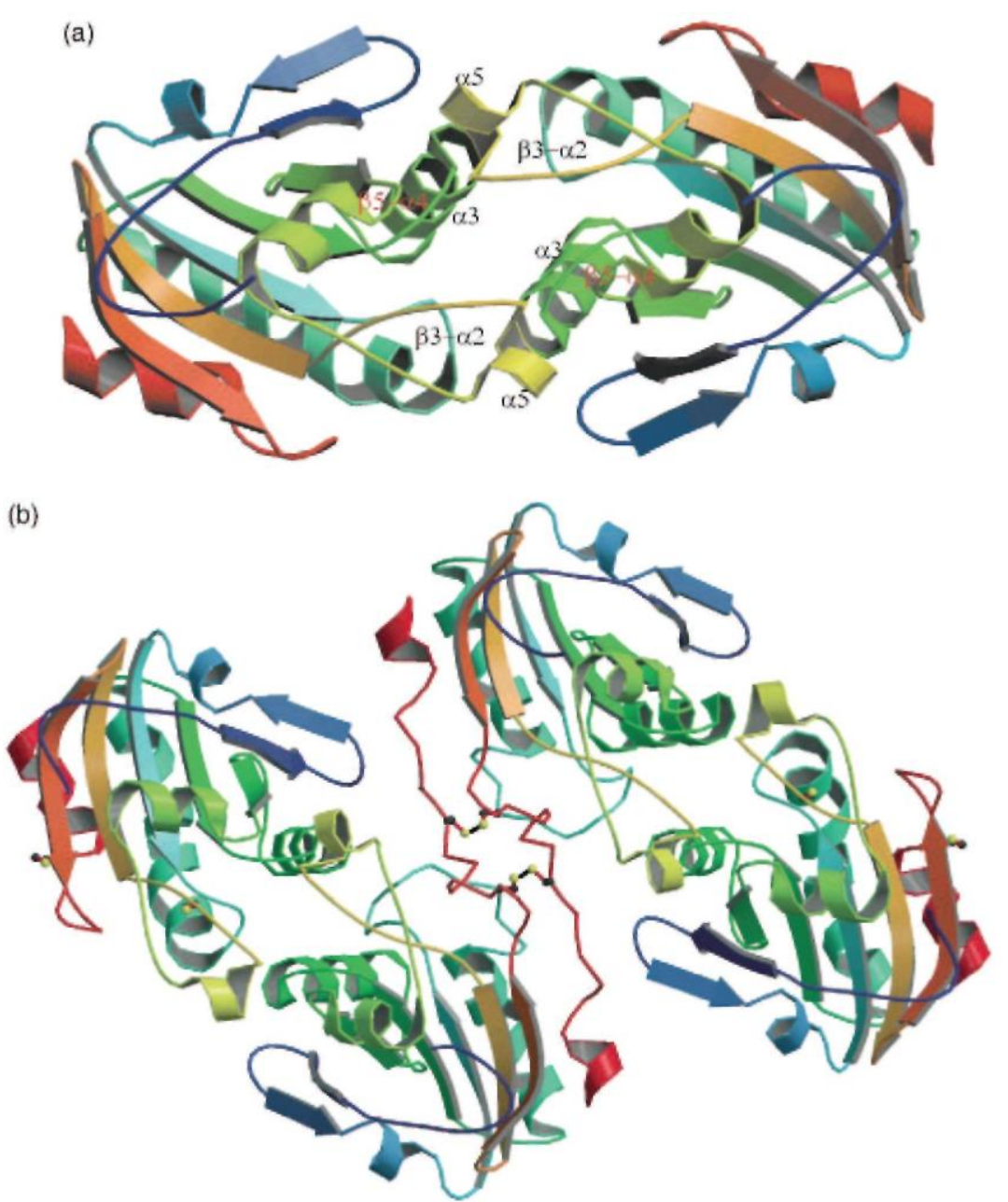


\section{MATERIALS AND METHODS}

\section{Crystallization}

The expression and purification of recombinant human PRDX5 have been described. ${ }^{18,22}$ The crystals of the $\mathrm{His}_{6}$-tagged molecule were grown under reducing conditions by hanging-drop, vapor-diffusion at $291 \mathrm{~K}$ with the well solution consisting of $20 \%$ (w/v) polyethylene glycol (PEG) 3350 as precipitant, $0.1 \mathrm{M}$ sodium citrate buffer (pH 5.3), $1 \mathrm{mM}$ 1,4-dithio-DL-threitol (DTT) as reductant and $0.02 \%(\mathrm{w} / \mathrm{v})$ sodium azide. The hangingdrop was formed by mixing $2 \mu \mathrm{l}$ of the protein solution $\left(10 \mathrm{mg} \mathrm{ml}^{-1}\right)$ with $2 \mu 1$ of the well solution. Crystals with typical dimensions of $0.15 \mathrm{~mm}$ appeared after about five days and deteriorated a few days later. It was suggested that this degradation could be the result of the oxidation of the protein molecule after the growth of the crystal. This oxidation could result in some conformational changes incompatible with the crystal packing.

\section{Data collection, structure determination and refinement}

Before data collection, the crystals were cryo-soaked in a solution identical with the mother liquor but containing $15 \%(\mathrm{v} / \mathrm{v})$ glycerol and flash-cooled at $100 \mathrm{~K}$. The data were collected on beam-line BW7B at EMBL c/o Desy (Hamburg, Germany) using an MAR345 imaging-plate detector. A resolution of $2.0 \AA$ was achieved using a wavelength of $0.8441 \AA$. The crystals are orthorhombic, space group $\mathrm{C} 222_{1}$, with $a=79.20 \AA \AA$, $b=102.05 \AA$, $c$ $=145.06 \AA$. The volume of the unit-cell suggests the presence of three polypeptide chains in the asymmetric unit $\left(V_{\mathrm{M}}{ }^{32}=2.89 \AA^{3} / \mathrm{Da}\right)$. All the measurements were indexed and integrated using the programme DENZO ${ }^{33}$ and merged with the programme SCALEPACK. ${ }^{33}$ Statistics of data collection and processing are given in Table 1. The presence of non-crystallographic symmetry (NCS) was investigated using the programme MOLREP ${ }^{34}$ of the $^{-1}$ CCP4 suite $^{35}$, applied to data comprised between 7.0 ̊ and 4.0 ̊́. This resulted in the clear indication of an NCS 2-fold axis corresponding to the polar coordinates $\left(\theta=90^{\circ}, \varphi=38.5^{\circ}, \chi=180^{\circ}\right)$ and thus located in the $(a, b)$ plane. Furthermore, a Patterson function computed at a resolution of $3.5 \AA$ did not indicate the presence of a pure translation between NCS related chains. These results suggest the presence of only two polypeptide chains in the asymmetric unit, but this situation would correspond to a more unlikely value of $V_{M}=4.34 \AA^{3} / \mathrm{Da}$. The resolution of the crystal structure was attempted by the molecular replacement method using the programme $\mathrm{AMoRe}^{36}$ applied to data between $20.0 \AA \hat{\text { and }} 3.5 \AA$ and the structure 1 hd 2 as a model. Looking for two independent polypeptide chains, two different solutions with similar figures of merit were found. One of the two chains present in the two solutions was the same while the remaining one was different. An examination of the packing with the programme $\mathrm{O}^{37}$ showed that, in both cases, large cavities were present in which the remaining chain could fit perfectly, indicating the presence of three polypeptide chains in the asymmetric unit, which will be labelled A, B and C. An analysis of the relationship between the three chains shows that the pairs A-B and A$\mathrm{C}$ are both related by the non-crystallographic 2 -fold axis at $\left(\theta=90^{\circ}, \varphi=38.5^{\circ}, \chi=180^{\circ}\right)$. The chains $\mathrm{B}$ and $\mathrm{C}$ are thus more or less parallel with each other but with a slight inclination preventing the apparition of a pure translation peak in the Patterson function.

The structure was refined using the programme REFMAC $5^{38}$ of the CCP4 suite. ${ }^{35}$ A rigid body refinement applied to data between $20 \AA$ and $2.75 \AA$ provided an R-value of $33.2 \%$. At this stage, a visual examination with the programme $\mathrm{O}^{37}$ revealed that chains $\mathrm{A}$ and $\mathrm{B}$ were very similar to the model of the reduced form (lhd2) used for the molecular replacement and required only minor adjustments. On the other hand, in chain $\mathrm{C}$ it was necessary to retrace two fragments corresponding to residues 46-52 and 144-161. These two fragments contain the two cysteine residues (Cys47 and Cys151) supposed to form an intramolecular disulfide bond in the oxidized form. A disulfide bond is indeed observed but it is intermolecular between two symmetry-related polypeptide chains C. The details are described in Discussion. The N-terminal His ${ }_{6}$ tag and the linker connecting the His tag $_{6}$ to the protein were not observed except for one residue (SerO) in chain A. As it was also the case in the previous reduced crystal forms of PRDX5, ${ }^{22,31}$ a benzoate ion is observed close to the cleft containing the active-site Cys47 in the two reduced chains (A and B) but is not present in the oxidized chain C. A total of 62 ordered solvent molecules was incorporated by the programme ARP/wARP ${ }^{39}$ During the final steps, the hydrogen atoms were incorporated in riding positions and the mean-square displacements of rigid bodies were refined, each polypeptide chain being defined as a different TLS group. The final $R$ value is $0.221\left(\mathrm{R}_{\text {free }}=0.259\right)$ for all available data to a resolution of $2.0 \AA$. The final statistics of the refinement are given in Table 1 . 


\section{SDS-PAGE analysis}

Electrophoresis of recombinant PRDX5 protein was carried out in SDS-12\% (w/v) polyacrylamide gels. ${ }^{40}$ PRDX5 $(10 \mu \mathrm{g})$ was incubated either with increasing concentrations of $\mathrm{H}_{2} \mathrm{O}_{2}$ for one hour at $37{ }^{\circ} \mathrm{C}$ or with increasing concentrations of DTT for five minutes at room temperature followed by five minutes at $95{ }^{\circ} \mathrm{C}$. Several samples were also incubated with $\mathrm{N}$-ethylmaleimide $(10 \mathrm{mM})$ for 30 minutes at $37{ }^{\circ} \mathrm{C}$ to block free thiol groups in oxidized PRDX5 before SDS-PAGE analysis. Proteins were then subjected to non-reducing SDSPAGE and stained with Coomasie brilliant blue G-250.

\section{Gel filtration analysis}

Recombinant PRDX5 in $20 \mathrm{mM}$ Tris-HCl buffer (pH 7.5) containing $150 \mathrm{mM} \mathrm{NaCl}$ and with $10 \mathrm{mM}$ DTT (reducing conditions) or without DTT (non-reducing conditions) was applied onto an analytical Superdex 200 column (HR 10/30; Amersham Pharmacia Biotech) using an Amersham Pharmacia AKT ̈̈ Prime system. As previously indicated for SDS-PAGE analysis, samples were also treated with $N$-ethylmaleimide (10 mM) to block free thiol groups under non-reducing as well as reducing conditions. Analyses were performed at a flow rate of $0.4 \mathrm{ml} /$ minute with monitoring by measurement at $\mathrm{A}_{280}$.

\section{Mass spectrometry analysis}

Proteins in the gel were excised using a $1 \mathrm{~mm}$ sample corer (Fine Science Tools Inc.). Excised gel pieces were placed in $1.5 \mathrm{ml}$ polypropylene Eppendorf tubes and washed twice in $50 \mu \mathrm{l}$ of $50 \mathrm{mM} \mathrm{NH}_{4} \mathrm{HCO}_{3}$. The gel pieces were destained and dehydrated with $50 \mu \mathrm{l}$ of $50 \mathrm{mM} \mathrm{NH}_{4} \mathrm{HCO}_{3}, 50 \%(\mathrm{v} / \mathrm{v}) \mathrm{CH}_{3} \mathrm{CN}$ and then dried in a centrifugal evaporator. Enzymatic digestion was performed by the addition of $10 \mu \mathrm{l}$ of trypsin $\left(0.02 \mu \mathrm{g} \mu \mathrm{l}^{-1}\right)$ (Promega Madison, WI, USA) in $25 \mathrm{mM} \mathrm{NH}_{4} \mathrm{HCO}_{3}$ to each gel piece, followed by overnight incubation at 37 ${ }^{\circ} \mathrm{C}$. For MALDI mass spectrometry, a $0.5 \mu \mathrm{l}$ aliquot of the digestion supernatant was spotted onto a sample plate with $0.5 \mu \mathrm{l}$ of matrix $\left(5 \mathrm{mg} \mathrm{ml}^{-1} \alpha\right.$-cyano-4-hydro-xycinnamic acid and $0.5 \mathrm{pmol} \mu \mathrm{l}^{-1}$ renin as internal standard in $25 \%(\mathrm{v} / \mathrm{v})$ ethanol, $25 \%(\mathrm{v} / \mathrm{v})$ acetonitrile, $0.05 \%(\mathrm{v} / \mathrm{v})$ trifluoroacetic acid) and allowed to air dry. MALDI mass spectrometry was performed using a Micromass Maldi ${ }^{\mathrm{TM}}$ spectrometer (Manchester, UK) equipped with a 337 $\mathrm{nm}$ nitrogen laser. The instrument was operated in the positive reflectron mode at $20 \mathrm{kV}$ accelerating voltage with time lag focusing. Spectra were internally calibrated using the renin peptide. An initial mass tolerance of 50 ppm was used in all searches.

\section{Protein Data Bank accession numbers}

Final coordinates and structure factors have been deposited with the RCSB Protein Data Bank under accession numbers loc3 and rloc3sf, respectively.

\section{Acknowledgements}

This work was supported by grants from the Fonds National de la Recherche Scientifique (Belgium) and the Communauté française de Belgique-Action de Recherches Concertées. We also thank the European Community for Access to Research Infrastructure Action of the Improving Human Potential Programme to the EMBL Hamburg Outstation, contract number HPRI-1999-CT-00017. We thank Fabio Lucaccioni for technical assistance in gel filtration analysis. We are grateful to the members of the scientific staff at the EMBL-Hamburg outstation for their help during data collection. PS. and R.W. are research associates of the Belgian National Funds for Scientific Research.

\section{References}

1. Butterfield, L. H., Merino, A., Golub, S. D. \& Shau, H. (1999). From cytoprotection to tumor suppression: the multifactorial role of peroxiredoxins. Antioxid. Redox Signal. 1, 385-402.

2. Rhee, S. G., Kang, S. W., Chang, T. S., Jeong, W. \& Kim, K. (2001). Peroxiredoxins, a novel family of peroxidases. IUBMB Life, 52, 3541. 
3. Fujii, J. \& Ikeda, Y. (2002). Advances in our understanding of peroxiredoxin, a multifunctional, mammalian redox protein. Redox Rep. 7 , $123-130$.

4. Hofmann, B., Hecht, H.-J. \& Flohe, L. (2002). Peroxiredoxins. Biol. Chem. 383, 347-364

5. Wood, Z. A., Schroder, E., Harris, J. R. \& Poole, L. B. (2003). Structure, mechanisms and regulation of peroxiredoxins. Trends Biochem. Sci. $28,32-40$

6. Chae, H. Z., Chung, S. J. \& Rhee, S. G. (1994). Thioredoxin-dependent peroxide reductase from yeast, J. Biol. Chem. 269, 27670-27678

7. Chen, J.-W., Dodia, C, Feinstein, S. I., Jain, M. K. \& Fisher, A. B. (2000). 1-Cys peroxiredoxin, a bifunc-tional enzyme with glutathione peroxidase and phospholipase A2 activities, f. Biol. Chem. 275, 28421-28427.

8. Flohe, L., Hecht, H. J. \& Steinert, P. (1999). Glutathione and trypanothione in parasitic hydroperoxide metabolism. Free Radic. Biol. Med. 27, 966-984.

9. Li Calzi, M. \& Poole, L. B. (1997). Requirement for the two AhpF cystine disulfide centers in catalysis of peroxide reduction by alkyl hydroperoxide reductase. Biochemistry, 36,13357-13364.

10. Lee, S. P., Hwang, Y. S., Kim, Y. J., Kwon, K. S., Kim, H. J., Kim, K. \& Chae, H. Z. (2001). Cyclophilin A binds to peroxiredoxins and activates its peroxidase activity, J. Biol. Chem. 276, 29826-29832.

11. Wood, Z. A., Poole, L. B. \& Karplus, P. A. (2003). Peroxiredoxin evolution and the regulation of hydrogen peroxide signaling. Science, $300,650-653$.

12. Seo, M. S., Kang, S. W., Kim, K., Baines, I. C, Lee, T. H. \& Rhee, S. G. (2000). Identification of a new type of mammalian peroxiredoxin that forms an intramolecular disulfide as a reaction intermediate. J. Biol. Chem. 275, $20346-20354$.

13. Shen, C. \& Nathan, C. (2002). Nonredundant antioxidant defense by multiple two-cysteine peroxiredoxins in human prostate cancer cells. Mol. Med. 8, 95-102

14. Pak, J. H., Manevich, Y., Kim, H. S., Feinstein, S. I. \& Fisher, A. B. (2002). An antisense oligonucleotide to 1-cys peroxiredoxin causes lipid peroxidation and apoptosis in lung epithelial cells, J. Biol. Chem. 277, 49927-49934.

15. Lee, T. H., Kim, S. U., Yu, S. L., Kim, S. H., Park, D. S., Moon, H. B. et al. (2003). Peroxiredoxin II is essential for sustaining life span of erythrocytes in mice. Blood, 101, 5033-5038.

16. Wang, X., Phelan, S. A., Forsman-Semb, K., Taylor, E. F., Petros, C., Brown, A. et al. (2003). Mice with targeted mutation of peroxiredoxin 6 develop normally but are susceptible to oxidative stress, J. Biol. Chem. 278, 25179-25190.

17. Neumann, C. A., Krause, D. S., Carman, C. V., Das, S., Dubey, D. P., Abraham, J. L. et al. (2003). Essential role for the peroxiredoxin Prdx1 in erythrocyte antioxidant defence and tumour suppression. Nature, 424, 561-565.

18. Knoops, B., Clippe, A., Bogard, C, Arsalane, K., Wattiez, R., Hermans, C. et al. (1999). Cloning and characterization of AOEB166, a novel mammalian antioxidant enzyme of the peroxiredoxin family. J. Biol. Chem. 274, 30451-30458.

19. Kropotov, A., Sedova, V., Ivanov, V., Sazeeva, N, Tomilin, A., Krutilina, R. et al. (1999). A novel human DNA-binding protein with sequence similarity to a subfamily of redox proteins which is able to repress RNA-polymerase-III-driven transcription of the Alu-family retroposons in vitro. Eur. J. Biochem. 260, 336-346.

20. Yamashita, H, Avraham, S., Jiang, S., London, R., Van Veldhoven, P. P., Subramani, S. et al. (1999). Characterization of human and murine PMP20 peroxisomal proteins that exhibit antioxidant activity in vitro. J. Biol. Chem. 274, 29897-29904.

21. Zhou, Y, Kok, K. H, Chun, A. C. S., Wong, C. M., Wu, H. W., Lin, M. C. M. et al. (2000). Mouse peroxiredoxin V is a thioredoxin peroxidase that inhibits p53-induced apoptosis. Biochem. Biophys. Res. Commun. 268, 921-927.

22. Declercq, J.-P, Evrard, C, Clippe, A., Vander Stricht, D., Bernard, A. \& Knoops, B. (2001). Crystal structure of human peroxiredoxin 5,a novel type of mammalian peroxiredoxin at 1.5 A resolution, $f$. Mol. Biol. 311, 751-759.

23. Laskowski, R. A., MacArthur, M. W., Moss, D. S. \& Thornton, J. M. (1993). PROCHECK: a program to check the stereochemical quality of protein structures. J. Appl. Crystallog. 26, 283-291.

24. Martin, J. F. (1995). Thioredoxin-a fold for all reasons. Structure, 3, 245-250.

25. Hirotsu, S., Abe, Y, Okada, K., Nagahara, N., Hori, H., Nishino, T \& Hakoshima, T (1999). Crystal structure of a multifunctional 2-Cys peroxiredoxin heme-binding protein 23 kDa/proliferation-associ-ated gene product. Proc. Natl Acad. Sci. USA, 96, $12333-12338$.

26. Schröder, E., Littlechild, J. A., Lebedev, A. A., Errington, N, Vagin, A. A. \& Isupov, M. N. (2000). Crystal structure of decameric 2-Cys peroxiredoxin from human erythrocytes at 1.7 A resolution. Structure, 8, 605-615. 
27. Kim, S. J., Woo, J. R., Hwang, Y S., Jeong, D. G., Shin, D. H., Kim, K. \& Ryu, S. E. (2003). The tetrameric structure of Haemophilus influenza hybrid-Prx5 reveals interactions between electron donor and acceptor proteins, J. Biol. Chem. 278, 10790-10798.

28. Choi, H. J., Kang, S. W., Yang, C. H, Rhee, S. G. \& Ryu, S. E. (1998). Crystal structure of a novel human peroxidase enzyme at $2.0 \AA$ resolution. Nature Struct. Biol. 5, 400-406.

29. Alphey, M. S., Bond, C. S., Tetaud, E., Fairlamb, A. H. \& Hunter, W. N. (2000). The structure of reduced tryparedoxin peroxidase reveals a decamer and insight into reactivity of 2Cys-peroxiredoxins. f. Mol. Biol. 300, 903-916.

30. Wood, Z. A., Poole, L. B., Hantgan, R. R. \& Karplus, P. A. (2002). Dimers to doughnuts: redox-sensitive oligomerization of 2-cysteine peroxiredoxins. Biochemistry, 41, 5493-5504.

31. Declercq, J.-P. \& Evrard, C. (2001). A twinned mono-clinic crystal form of human peroxiredoxin 5 with eight molecules in the asymmetric unit. Acta Crystal-log, sect. D, 57, 1829-1835.

32. Matthews, B. W. (1968). Solvent content of protein crystals, J. Mol. Biol. 33, 491-497.

33. Otwinowski, Z. \& Minor, W. (1997). Processing of X-ray diffraction data collected in oscillation mode. Methods Enzymol. 276, 307326.

34. Vagin, A. \& Teplyakov, A. (1997). MOLREP: an automated program for molecular replacement, f. Appl. Crystallog. 30, $1022-1025$.

35. Collaborative Computational Project, Number 4 (1994). The CCP4 suite: programs for protein crystallography. Acta Crystallog. sect. D, $50,760-763$

36. Navaza, J. (1994). AMoRe: an automated package for molecular replacement. Acta Crystallog. sect. A, 50, 157-163.

37. Jones, T. A., Zou, J.-Y., Cowan, S. W. \& Kjeldgaard, M. (1991). Improved methods for building protein models in electron-density maps and the location of errors in these models. Acta Crystallog. sect. A, 47, 110-119.

38. Murshudov, G. N., Vagin, A. A. \& Dodson, E. J. (1997). Refinement of macromolecular structures by the maximum-likelihood method. Acta Crystallog. sect. D, 53, 240-255.

39. Perrakis, A., Morris, R. M. \& Lamzin, V. S. (1999). Automated protein model building combined with iterative structure refinement. Nature Struct. Biol. 6, 458-463.

40. Laemmli, U. K. (1970). Cleavage of structural proteins during the assembly of the head of bacteriophage T4. Nature, $227,680-685$.

41. Kraulis, P. J. (1991). MOLSCRIPT: a program to produce both detailed and schematic plots of protein structures, J. Appl. Crystallog. 24, 946-950

42. Merritt, E. A. \& Bacon, D. J. (1997). Raster3D: photorealistic molecular graphics. Methods Enzymol. 277, 505-524.

43. Nicholls, A., Bharadway, R. \& Honig, B. (1993). GRASP: graphical representation and analysis of surface properties. Biophys. f. 64, $166-170$. 